\title{
Workshop on proposal writing on research for health care professionals: a brief report
}

This article was published in the following Dove Press journal:

Journal of Multidisciplinary Healthcare

Sunil Shrestha ${ }^{1,2}$
Krisha Danekhu ${ }^{2}$
Neeraj Sharma ${ }^{3}$
Praval Khanal
Safiur Rahman Ansari ${ }^{5}$
Sudip Shrestha ${ }^{6}$
Rano Mal Piryani ${ }^{7}$
Mohamed Izham
Mohamed Ibrahim

University, Doha, Qatar
Correspondence: Sunil Shrestha Clincial Pharmacist and Research Associate, Nepal Cancer Hospital and Research Center Pvt. Ltd., Harisidhhi, Lalitpur, Nepal

Tel +977 I 9851220488

Email sunil.shrestha@nch.com.np; sunilcresta@gmail.com

\begin{abstract}
A one-day workshop on proposal writing for research for health care professionals was organized by Hospital Research Board (HRB), Nepal Cancer Hospital and Research Center Pvt. Ltd, Harisiddhi, Lalitpur, Nepal on 2nd March 2019. The main aim of this workshop was to identify, motivate and prepare health care professionals for conducting research in their respective professional institution in collaboration. The workshop was facilitated by international and national resource persons. The deliberations of the workshop were focused on seven topics: "Turning research into impact, Essentials of the research protocol, Why proposals are rejected?, Plagiarism in medical research, Research with medical records, Grant writing workshop, Manuscript writing workshop" based on a presentation by the different resource persons. Ninety-nine persons participated in the workshop including physicians, medical oncologists, pharmacists, nurses and other allied health professionals. The interactive teaching-learning methods were utilized in all sessions of the workshop. The feedback of the participants was taken on semi-structured feedback format. Overall evaluations from the feedback forms showed that majority $(90 \%)$ of the participants agreed that the workshop achieved its objectives with a major recommendation as to the allocation of short duration for the workshop and timely organize of research activities regarding scientific writing. In conclusion, the workshop on research proposal writing for health care professionals was successfully organized and the participants are looking forward for future ones.
\end{abstract}

Keywords: workshop, proposal writing, research, health care professionals; Nepal

\section{Introduction}

Nepal is a country with huge possibilities in the field of health-related research. Studies have reported the high availability of pharmaceutically important medicinal plants ranging from plain part to the upper Himalayas in Nepal. ${ }^{1-3}$ Similarly, the prevalence of some of the communicable and non-communicable diseases is also comparative. ${ }^{4-6}$ Although there are limited number of studies that have investigated the diseases and control strategies in different perspectives, there is more need of such studies from both governmental and non-governmental organizations. The presence of this scenario in Nepal suggests that there are enormous possibilities of research in the areas of pharmaceutical products, epidemiology, agricultural biology and so on. Despite the possibilities of different kinds of health-related research and the presence of many health professionals involved in various fields, ${ }^{7}$ there is a lag in the scientific publication from those areas. One of the major contributing factors for having no or less publication is due to a lack of knowledge of scientific writing such as manuscript writing and grant writing. Other possible 
factors could be poor health research plantings, lack of proper training and underdeveloped research culture. ${ }^{8}$

With this background, a workshop was organized with the international and national resource persons with the aim to improve the present knowledge on scientific writing in health researchers and to build the concept of academic writing to the new researchers. Therefore, the following section of the article would mainly follow as the brief report of the workshop event.

\section{Brief report}

A Workshop on Proposal Writing on research for Health Care Professionals was organized on 2nd March 2019 in the conference hall, Nepal Cancer Hospital and Research Center (NCHRC), Harisiddhi, Lalitpur, Nepal, by Hospital Research Board (HRB), NCHRC. Organizing and conducting such kind of workshops are more common in health and other science areas. ${ }^{9,10}$

\section{Objectives of the workshop}

The objectives of the workshop were:

- To identify, motivate and prepare health care professionals and academicians to conduct research with collaboration in their respective professional institutions;

- To write a research grant;

- To discuss how to translate research into impact;

- To discuss the essentials of a research protocol;

- To write a manuscript for a scientific journal;

- To avoid plagiarism in medical writing;

- To motivate participants for multidisciplinary research.

The participants were from a different background of the health care profession. They include physicians, medical oncologists, pharmacists, nurses and other allied health professionals.

The event was inaugurated with a welcome speech by Dr Sudip Shrestha, Executive Chairman of NCHRC, followed by insight by Medical Director, Dr Bijesh Raj Ghimire and an overall overview of the workshop by $\mathrm{Dr}$ Sunil Shrestha, Program Coordinator of the event. At the end of the event, a vote of thanks was delivered by $\mathrm{Dr}$ Murari Man Shrestha, Member Secretary, HRB, NCHRC. Nepal Health Research Council assigned 5 Credit Hour Certificates and were distributed to all participants.

International resource person, Prof. Dr Mohamed Izham Mohamed Ibrahim, Professor of Social and Administrative
Pharmacy, Head of Research Graduate Studies - Pharmacy in Qatar University conducted a session on "Turning research into impact" and "Why proposals are rejected?." He also facilitated mini-workshop on "Grant Writing" and "Manuscript Writing." Prof. Dr Rano Mal Piryani, Consultant Internal Medicine, Chest Medicine and Medical Education and Resource person for training in Health Professions Education, Bioethics and Medical Research, Professor of Internal Medicine, Chief Coordinator Health Professions Training Committee in Universal College of Medical Sciences, Bhairahawa, Nepal, conducted a session on "Essentials of Research Protocol." Dr. Praval Khanal from Manchester Metropolitan University, United Kingdom discussed on "Plagiarism in Medical Research" while Dr. Safiur Rahman Ansari from HELF Informatics Pvt Ltd put light on "Research with Medical Records."

\section{Key points of sessions Turning research into impact}

The main points were on how to utilize research outcomes and the importance of communication activities and strategies to turn research output into impact. The speaker had also touched on the use of knowledge to solve public health problems stating from the different types of research. He explained the need for effective communication activity necessity to enhance research utilization at each level followed by improvement of the way of conveying evidence-based research to policy-makers and to bridge the gap between policy and evidence.

\section{Essentials of research protocol}

This session was focused thoroughly on the different components of the research protocol. The research protocol is a science and art of describing intended outcomes, practices, process and procedures of a research study. The resource person deliberated on how components of the research protocol vary depending on the discipline and objectives of the study. He successfully explained 12 main components of a research protocol:

- Abstract (overview of the contents);

- Literature review or background (overview of research done \& rationale for conducting present study);

- Research question follows PICOTS and FINER frameworks (PICOTS: Population, Intervention, Comparison, Outcome, Time and Settings) (FINER: Feasible, Interesting, Novel, Ethical, Relevant); 
- Research outcomes (primary \& secondary outcomes or endpoints);

- Study design and research approach (population of interest, inclusion/exclusion criteria, study settings, sampling strategy and sample size);

- Data extraction or collection methods (ethical approval, informed consent, data collection tool and data collection process);

- Data management policies (data entry, data coding, privacy and confidentiality);

- Statistical procedures (intended statistical tools to be applied for analysis and interpretation of data);

- Risks to subjects (informed consent, disclosure of information, issue of vulnerability of participants);

- Threats to validity (confounding variables, sample size limitation, drop out);

- Works cited (references);

- Appendices (survey measures, interview guide, etc.).

He stated the importance of understanding the steps to develop a research protocol to conduct study appropriately and obtain reliable results.

\section{Why proposals are rejected?}

The resource person in this session provided insight into the different reasons for rejection of a research proposal during grant application. He explained that a research proposal is a document that informs others the proposed piece of research. Unsuitability and poorly designed proposals were described as the primary reasons for the rejection of the research proposal.

\section{Plagiarism in medical research}

This session was primarily focused on clarifying how serious is the plagiarism in medical research and how it can be avoided during article writing. The resource person explained the plagiarism is presenting other's ideas as our own work without giving credit to the main author followed by stating it as serious scientific misconduct. The resource person elaborated about the strategies that can be used for avoiding the plagiarism - explaining about the importance of paraphrasing, using a quote for direct sentence citing appropriately, and use of available plagiarism checker software.

\section{Research with medical records}

This session puts an idea about the importance of using Electronic Health Record (EHR) for the health-related research. Primarily, the resource person explained how the data can be collected during the routine delivery of health care in electronic format which is designed for observational research, safety surveillance, clinical research and regulatory uses. He mentioned that pre-determined queries, simple customizable queries and advanced customizable queries are used in the process of data extraction. In addition to those, he explained the importance of SQL language and its use for storing, manipulating and retrieving data in relational database management. SQL with script transforms allows to write commands that run the statistical procedure. Statistical scripts are embedded into Electronic Medical Record (EMR) and other software which analyses easily without loading heavy data files with high-speed execution. The speaker is experienced with EMR and later on pointed the challenges with EMR as:

- the quality of data and its validation,

- timely access to electronic health record data,

- heterogeneity between systems,

- data security and privacy,

- system capabilities.

\section{Grant writing workshop}

This session was made interactive by providing a redacted part of the article to all the participants. The preparatory time was provided to the participants followed by interacting with them to construct the possible general and specific objectives of the study. The comparison was done between the suggested objectives and the authors' objectives mentioned in the study. The speaker discussed the possible methodology for the project based on the suggested objectives of the study.

\section{Manuscript writing workshop}

The content that is to be included in the manuscript was discussed. It started with the idea that how the writing should follow based on the objectives stated and the results of the study. The session provided the new and innovative idea about how the writing can be made easy for an author starting with the different components of the article rather than the regular sequence found in an article. At one point of the session, it was discussed that the manuscript writing can be made easier while writing in the order: results, methods, introduction, discussion, conclusion, abstract, references list, appendix, title page and cover letter. 


\section{Statistical analysis}

Descriptive analysis was performed to evaluate the effectiveness of the workshop. The feedback answers were entered in the MS-Excel 2010 (Microsoft Corporation, Redmond, WA, USA) initially and later exported to SPSS Version 21 (SPSS Inc., Chicago, IL, USA) and analysis was performed.

\section{Feedback on the workshop}

Feedback of the participants was taken on semi-structured questionnaire. The effectiveness of the workshops is usually measured by obtaining the feedback from the attendees. This process is applied by several previous studies. ${ }^{11,12}$ In the present workshop, a feedback questionnaire was provided to each participant of the workshop immediately after the completion of the workshop. (Appendix I).

\section{Major quantitative analysis results}

The questionnaire included several questions regarding the workshop ranging from the satisfaction of the contents of the workshop to the venue of the program. The overall response rates from the total attendee $(n=99)$ were $60.6 \%(n=60)$. Mean of overall assessment and comment on the organization of the event was 3.93 and 4.22 respectively (Table S1). The frequency of participants and their feedback on worthiness of individual topics was shown in Table S2.

Out of 60 participants of the workshops, $90 \%$ responded that the objectives of the workshop were obtained. $10 \%$ did not think that the objectives were met and mentioned about the time limitations as the crucial factor for their response (Table S3). Forty-one (68.33\%) responded they gained adequate knowledge and information, while $17(28.33 \%)$ mentioned that they got "somewhat" -meaning they build up the research concept (Table S4). Thirty-nine participants $(65.0 \%)$ responded that workshop was "Definitely" useful, while $25 \%$ mentioned it was "mostly" useful (Table S5).

Around 57\% rated the organization of the workshop at 3 (good) on Likert scale 1-5 (5= excellent, $4=$ very good, $3=$ good, $2=$ poor, $1=$ very poor, while $31.66 \%$ rated at 4 as very good).

\section{Major qualitative analysis results Best feature of the workshop}

The responses from participants were categorized into four main themes. Four themes included - resource persons/speakers for the workshop, time management by resource persons and organizers of the workshop, the practical significance of the workshop and the overall management of the workshop by organizers. Overall, the participants of the workshop were enthusiastic about the quality of the workshop and the included topics.

\section{Dissatisfaction about the workshop}

Three categories were developed from the feedback provided by the attendee of the workshop. The three categories included in dissatisfaction about the workshop are the short length of the workshop, logistical/venue criticisms, fewer interactions/group discussions during workshops.

\section{Recommendations for improving forthcoming workshops}

The various recommendations were obtained for improving future workshops. The aim of obtaining the recommendations was to understand the participants' opinion about the workshops, particularly regarding resource persons, the content and objectives of workshop and organizers. Categories for this section were necessity of this kind of research workshops, more interaction during workshops with round table discussion, appreciations and demand for a longer duration for workshop.

The results are shown in Appendix II.

\section{Discussion}

The present workshop on proposal writing for health care professionals aimed to provide knowledge on scientific writing - primarily focusing on grant writing, appropriate manuscript writing, avoiding plagiarism in medical research, turning research into impact and essentials of research protocol, the reasons for proposal rejection and research with medical records. The results from feedback form showed that there is an improvement in the participant's knowledge after attending the workshop. The attendee felt that the contents are strongly applicable to their jobs or clinical practices and they are willing to apply in the workplace; however, few complained about the length of the workshop - demanding for more workshops in near future with more group discussion and long duration. The participants were from different health fields, the areas more prominent for the research; therefore, the authors believe the knowledge gained could be beneficial for the attendee in the future.

Our results showed that most of the participants were satisfied with the venue where the workshop was organized and the topics/contents covered during the workshop. As some of the results of this workshop showed 
that participants were less stratified with venue and food. They suggested making a large space for the future workshop and improving the quality of the food.

Nepal is a country with large opportunities for research and technology, so the authors believe that this kind of workshop is important in the near future as well. Conducting this kind of workshop could be helpful for researchers, academicians, and others in capacity building and enhance research culture.

\section{Conclusion}

Workshop on Research Proposal Writing for Health Care Professionals was successfully organized. It motivated the health care professionals and academicians for including research as part of their professional activities. The workshop also motivated the participants to adopt a multidisciplinary and collaborative approach for conducting research.

\section{Acknowledgments}

The author(s) acknowledge Dr. Subish Palaian, Associate Professor of Pharmacy Practice from Gulf Medical University for the concept of organizing a workshop at Nepal for health care professionals. The author(s) also acknowledge Dr. Bijesh Raj Ghimire, Medical Director of Nepal Cancer Hospital and Research Center and members of organizing committee of the workshop of for their valuable sugguestions and support.

\section{Disclosure}

The authors report no conflicts of interest in this work.

\section{References}

1. Taylor RS, Manandhar NP, Towers GH. Screening of selected medicinal plants of Nepal for antimicrobial activities. J Ethnopharmacol. 1995;46(3):153-159.

2. Luitel DR, Rokaya MB, Timsina B, Münzbergová Z. Medicinal plants used by the Tamang community in the Makawanpur district of central Nepal. J Ethnobiol Ethnomed. 2014;10(1):5. doi:10.1186/ 1746-4269-10-5

3. Acharya K, Chaudhary R, Vetaas O. Medicinal plants of Nepal: distribution pattern along an elevational gradient and effectiveness of existing protected areas for their conservation. Banko Janakari. 2009;19(1):16-22. doi:10.3126/banko.v19i1.2178

4. Mishra SR, Neupane D, Bhandari PM, Khanal V, Kallestrup P. Burgeoning burden of non-communicable diseases in Nepal: a scoping review. Global Health. 2015;11(1):32. doi:10.1186/s12992-0150119-7

5. Karki A, Tiwari BR. Prevalence of acute diarrhoea in Kathmandu valley. JNMA J Nepal Med Assoc. 2007;46(168):175-179.

6. Thapa S Recent status of major infectious diseases in Nepal. Paper presented at: proceedings of First International Conference on Infectious Diseases \& Nanomedicine (ICIDN)-2012; 2012; Kathmandu, Nepal. doi:10.1094/PDIS-11-11-0999-PDN

7. Simkhada PP, Baral YR, van Teijlingen ER. Health and medical research in Nepal: a bibliometric review. Asia Pac J Public Health. 2010;22(4):492-500. doi:10.1177/1010539510371020

8. Teijlingen PEv. PARI: strengthening health research in Nepal; 2015. Available from: http://soscbaha.org/downloads/lecture_series/lxxxiii/ lecture_lxxxiii.pdf. Accessed June 6, 2019.

9. Shankar P, Izham M, Piryani R, Subish P. A one day workshop on scientific writing: brief report. Australas Med J (Online). 2010;1 (4):267.

10. Jawaid SA. Proceedings of workshop on scientific writing and publishing organized by EMAME-DHA at Dubai (March 22-24, 2018). Pak J Med Sci. 2018;34(4):1034-1042. doi:10.12669/ pjms.344.16084

11. Goyal M, Misra D, Rajadhyaksha S, Singh Y, Goyal N, Ravindran V. Effectiveness of a 1-day workshop on scientific writing conducted by the Indian journal of rheumatology. Indian J Rheumatol. 2018;13 (2):117-120. doi:10.4103/injr.injr_36_18

12. Steinert Y, McLeod PJ, Liben S, et al. Writing for publication in medical education: the benefits of a faculty development workshop and peer writing group. Med Teach. 2008;30(8):e280-e285. doi:10.1080/01421590801965129 


\section{Supplementary materials}

\section{Appendix I}

\section{Evaluation by participants}

The following questionnaire was given to participants to evaluate the workshop.

Participant Name (optional): Date:

Job Title:

Years in present position: years

1) What is your overall assessment of the event? (1 = insufficient $-5=$ excellent)

$$
\begin{array}{lllll}
1 & 2 & 3 & 4 & 5
\end{array}
$$

2) Which topics or aspects of the workshop did you find most interesting or useful?

3) Did the workshop achieve the workshop objectives?

$$
\text { Yes No If no, why? }
$$

4) Knowledge and information gained from participation at this event?

Met your expectations Yes No Somehow Will be useful/applicable in my work definitely mostly Somehow Not at all

5) How do you think the workshop could have been made more effective?

6) Please comment on the organization of the event (from $1=$ insufficient to $5=$ excellent)

$$
\begin{array}{lllll}
1 & 2 & 3 & 4 & 5
\end{array}
$$

7) Comments and suggestions (including activities or initiatives you think would be useful, for the future)

8) Further comments or suggestions

9) What other improvements would you recommend in this workshop?

10) What did you like best about this workshop?

11) What did you like least about this workshop?

Are you interested in receiving other educational materials/workshops from or e-mail updates about this project? Yes No.

If so, please write your name, address, e-mail, phone number, and the subject(s) and grade level(s) you work with most.

\section{Appendix II \\ Quantitative analysis}

Table SI Overall assessment and comment on the organization of the event

\begin{tabular}{|l|l|l|}
\hline & $\begin{array}{l}\text { What is your overall } \\
\text { assessment of the } \\
\text { event? }(\mathbf{n}=59)\end{array}$ & $\begin{array}{l}\text { Please comment on } \\
\text { the organization of } \\
\text { the event }(\mathbf{n = 5 8})\end{array}$ \\
\hline $\begin{array}{l}\text { Mean (SD) } \\
\text { Median (IQR) }\end{array}$ & $3.93(0.691)$ & $4.22(0.622)$ \\
$4(4-4)$ & $4(4-5)$ \\
\hline
\end{tabular}

Table S2 Descriptive table showing the frequency of participants and their feedback on worthiness of individual topics (multiple responses)

\begin{tabular}{|l|l|l|}
\hline $\begin{array}{l}\text { Which topics or aspects of the } \\
\text { workshop did you find most inter- } \\
\text { esting or useful? }\end{array}$ & Frequency & Percent \\
\hline Essentials of research protocol & 21 & \\
Plagiarism in medical research & 24 & $45 \%$ \\
Grant writing workshop & 25 & $41.67 \%$ \\
Manuscript writing workshop & 24 & $40 \%$ \\
Why proposal are rejected? & 14 & $23.33 \%$ \\
Research with medical records & 14 & $23.33 \%$ \\
\hline
\end{tabular}

Table S3 Frequency showing the overall result of feedback on achievement of program objective and possible suggestions

\begin{tabular}{|l|l|l|}
\hline $\begin{array}{l}\text { Did the workshop achieve the work- } \\
\text { shop objectives? }\end{array}$ & Frequency & Percent \\
\hline $\begin{array}{l}\text { No } \\
\text { Yes } \\
\text { Total }\end{array}$ & 4 & 6.7 \\
\hline If no, why? & 54 & $\begin{array}{l}90.0 \\
100.0\end{array}$ \\
\hline $\begin{array}{l}\text { Didn't seem like a workshop as there was } \\
\text { no group separation }\end{array}$ & I & 1.7 \\
$\begin{array}{l}\text { No group discussion } \\
\text { Should be more basic \& related to } \\
\text { oncology }\end{array}$ & I & 1.7 \\
Time limitation & & 1.7 \\
\hline
\end{tabular}


Table S4 Frequency of participants reporting the knowledge gained after the events

\begin{tabular}{|l|l|l|}
\hline $\begin{array}{l}\text { Knowledge and information gained } \\
\text { from participation at this event? }\end{array}$ & Frequency & Percent \\
\hline Not answered & $\mathrm{I}$ & 1.66 \\
Met your expectations & $\mathrm{I}$ & 1.66 \\
Somehow & 17 & 28.33 \\
Yes & 41 & 68.33 \\
Total & 60 & \\
\hline
\end{tabular}

Table S5 Descriptive table showing the frequency of participants and their feedback on the worthiness of overall workshop

\begin{tabular}{|l|l|l|}
\hline Workshop is useful & Frequency & Percent \\
\hline Not Answered & $\mathrm{I}$ & 1.66 \\
Definitely & 39 & 65 \\
Mostly & 15 & 25 \\
Somehow & 4 & 6.66 \\
Will be useful/applicable in my work & $\mathrm{I}$ & 1.66 \\
Total & 60 & \\
\hline
\end{tabular}

\section{In-depth qualitative analysis category \\ Best feature of the workshop}

For the responses to these questions, four categories are developed from our coding. The participants of the workshop seemed to be enthusiastic about the quality of the workshop and its contents.

\section{- 1. Resource person/Speaker of the workshop}

"Expert Advice Dr. Izham's Sessions", "Good tutor", "Familiar Interaction by resource persons", "Prof Izham Breakthrough skills," etc., are things wrote by the participants of the workshop. They also acknowledge that they like sessions.

- 2. Time management by resource persons and organizers

Some of the participants like the time management by resource persons along with organizers. Participants wrote that "Excellent Time management", "Everything was on time".

- The practical significance of the workshop

Participants cherished techniques that were "Helpful in future research work" and helped them with "manuscript writing" and "grant writing". Many participants wrote that they gained knowledge in different aspects of proposal writing.

- The event management of the workshop by the organizers

The participants were high in number in accordance with the size of the hall. However, participants expressed gratitude for organizing committee writing that "Well-Managed".

\section{Dissatisfaction about the workshop}

For the responses to these questions, three categories are developed from our coding.

- The short length of the workshop

As the workshop was conducted for one day only, most participants complained about the short duration of the workshop. One of the participants wrote", "Please have a two-three day of the workshop," another participant wrote that "Contents are too much but one day is not enough". Majority of participants were happy with the workshop but they dislike the length of the workshop.

\section{- Logistical/locations criticisms}

In this category, we label it as a minor criticism to the workshop or venue settings. One of the participants wrote that "Arrange big hall for future events" and another one wrote, "To have round table settings for discussion and group work". Similarly, one of the participants wrote that "Please invite fewer participants" and another one disliked the food of the workshop. One participant was unhappy with the registration system for the workshop and wrote: "Registration should be done via online".

- Fewer interactions/group discussion during workshops

As there was less group discussion during workshops, some of the participants were dissatisfied. Some participants of the workshop were dissatisfied with less practical base sessions on grant proposal writing and manuscript writing workshop. They wanted more practical-based sessions on such theme of manuscript writing and grant writing. 


\section{Recommendations for improving forthcoming workshops}

The different recommendations from participants were obtained for improving future workshops which will be organized. In these open-ended questions, our aim was to find out what recommendations participants might have as a way to offer feedback for resource persons, the content and objectives of workshop and organizers. Participants as an alternative appeared to use this open question to express their appreciation and demand for additional future workshop opportunities.

- Necessity of this kind of research workshops

Most of the participants were seeking for this kind of workshops or training. They are also seeking for chances to attend workshops in the future. As one participant wrote, "Please organize such of workshops". Participants are requesting organizers to organize such kind of programs in the future. Participants wrote that "Invite/Inform in next workshop", "Organize a workshop on SPSS".

"Need more similar class," "please organize more such workshops, seminars, and conferences especially on qualitative research, mixed research, plagiarism".

"Conduct periodically to enhance research skills," "keep providing such program," "looking forward for same kind of program," "more workshops in pharmacy for upgrading.
- More interactive during workshops with round table discussion

Most of the participants were dissatisfied with less interaction during workshops. One of the participants wrote to "keep fewer participants so that more interaction is possible" and another participant wrote "Please have round table discussion".

\section{- Appreciation}

Many participants of the workshop have one voice which stated gratitude for the experience of the workshop. Participants wrote, "Thank you for this workshop opportunity", "Thank you for the wonderful workshop", "I feel lucky to participate". Others expressed gratitude to resource persons and contents of the workshop" saying "Resource person have influential personality" Some of the participants wrote expressed gratitude to organizers saying" Excellent and well organized", "Well managed and organized systemically", "every session was on time", "perfect time management", etc.

- Demanded longer duration for workshop

As many of the participants were dissatisfied with a shorter duration for the workshop, they suggested organizing such kind of workshops with long duration.

\section{Publish your work in this journal}

The Journal of Multidisciplinary Healthcare is an international, peerreviewed open-access journal that aims to represent and publish research in healthcare areas delivered by practitioners of different disciplines. This includes studies and reviews conducted by multidisciplinary teams as well as research which evaluates the results or conduct of such teams or healthcare processes in general. The journal covers a very wide range of areas and welcomes submissions from practitioners at all levels, from all over the world. The manuscript management system is completely online and includes a very quick and fair peer-review system. Visit http://www.dovepress.com/testimonials. php to read real quotes from published authors. 